\title{
Association between pentraxin-3, body fat distribution and carotid intima media thickness in premenopausal obese women
}

\author{
Ozen Dedeoglu${ }^{1}$, Guzin Fidan-Yaylali ${ }^{2 \star}$, Duygu Herek ${ }^{3}$, Suleyman Demir ${ }^{4}$, Hande Senol ${ }^{5}$ and \\ Senay Topsakal ${ }^{2}$
}

${ }^{1}$ Department of Internal Medicine, Sırnak State Hospital, Sırnak, Turkey.

${ }^{2}$ Department of Endocrinology and Metabolism, Faculty of Medicine, Pamukkale University, Kinikli Campus, Denizli, 20070, Turkey.

${ }^{3}$ Department of Radiology, Faculty of Medicine, Pamukkale University, Kinikli Campus, Denizli, 20070, Turkey.

${ }^{4}$ Department of Biochemistry, Faculty of Medicine, Pamukkale University, Kinikli Campus, Denizli, 20070, Turkey.

${ }^{5}$ Department of Biostatistics, Faculty of Medicine, Pamukkale University, Kinikli Campus, Denizli, 20070, Turkey.

Accepted 12 December, 2018

\begin{abstract}
Pentraxin 3 (PTX3) is an acute-phase protein that shares structural homology with C-reactive protein (CRP). Because obesity and metabolic syndrome (MetS) are considered chronic inflammatory states, PTX3 might be involved in the pathogenesis of obesity and MetS as well as CRP. In this study, we aimed to investigate the relationships between PTX3, CRP, body fat distribution and carotid intima media thickness (CIMT). 73 obese premenopousal women and 53 women with normal body mass index (BMI) took part in this study. Body fat distribution was evaluated by ulrasonography and by Bioelectrical Impedance Analysis (BIA). PTX3 was similar in both groups. PTX3 was also similar in obese patients with and without insulin resistance. CRP is significantly higher in obese patients $(p<0.01)$. It was also significantly higher in insulin resistance obese patients $(p<0.01)$. There was no correlation between BMI and any of the inflamatory markers. Multiple regression analysis showed that PTX3 is positively correlated with waist circumference (WC) $(p=0.036$, beta $=+0.436)$ and negatively correlated with subcutaneous fat $(S C F)(p=0.011$, beta $=-0.310)$. In conclusion, although PTX3 levels were not higher in obese patients and have no correlation with visceral fat, PTX3 levels were correlated with WC. In premenoposal obese women PTX3 levels were not related to subclinical atherosclerosis measured by CIMT.
\end{abstract}

Keywords: Pentraxin-3, visceral fat, subcutaneous fat, atherosclerosis.

*Corresponding author. E-mail: guzinf@gmail.com. Tel: +90 258 2965753. Fax: +90 2582127792.

\section{INTRODUCTION}

Pentraxin-3 (PTX3) is an acute-phase protein, it has structural similarity with C-reactive protein (CRP). In response to inflammatory signals, the liver produces the classic short pentraxin CRP (Pepys and Hirschfield, 2003), which strongly associates with cardiometabolic disorders such as obesity, metabolic syndrome, diabetes mellitus, and atherosclerosis (Ridker et al., 2000). In contrast, a variety of immune and resident cells involved in cardiometabolic disorders - including macrophages, dendritic cells, fibroblasts, smooth muscle cells, endothelial cells, and adipocytes - secrete the long pentraxin PTX3 (Garlanda et al., 2005).

PTX3 plasma levels rapidly rise in the early phase after ischemic heart disorders (Peri et al., 2000; Bonacina et al., 2013). Because different cells within adipose tissue might produce PTX3 (Abderrahim-Ferkoune et al., 2003), 
it could be a more sensitive marker of inflammation caused by obesity than CRP. The association between circulating levels of PTX3 and measures of adiposity is inconsistent. Some studies have found positive associations (Alberti et al., 2009; Miyaki et al., 2010), whereas other have demonstrated inverse associations (Osorio-Conles et al., 2011; Barazzoni et al., 2013; Miyaki et al., 2013; Miyamoto et al., 2011; Ogawa et al., 2010; Yamasaki et al., 2009; Witasp et al., 2014).

PTX3 levels were not associated with early stage atherosclerosis measured by intima media thickness in the Bruneck, ARMY and ARFY studies (Kiechl et al., 2001; Knoflach et al., 2003; Knoflach et al., 2009). Knoflach et al. described a significant association of PTX3 with advanced human atherosclerosis. When compared to CRP, PTX3 predicted prevalent cardiovascular disease better, had fewer associations with other vascular risk conditions and may be more specific for vascular wall inflammation (Knoflach et al., 2012).

In this study, we aimed to investigate the relationships between fat distribution, carotid intima media thickness and PTX3, together with CRP in premenopausal obese women.

\section{MATERIALS AND METHODS}

73 premenopausal obese women (aged 17 to 55 years) and 53 women with normal BMI took part in this study. All women were required to be premenopausal, as evidenced by regular menstrual cycles. Exclusion criteria included diagnosis of polycystic ovary syndrome, regular exercise more than 2 hours per week, pregnancy, current breastfeeding, any disorders of glucose or lipid metabolism, use of medication that could affect body composition or glucose metabolism (including oral contraceptives, cholesterol medications, and blood pressure medications), current use of tobacco, presence of acute infection. Participants were informed of the experimental design, oral and written consents were obtained. This study was approved by the Medical Ethics Committee of the the participating university (the registry number 60116787020/41760)

\section{Anthropometric evaluation}

Obesity was defined as $\mathrm{BMI}>30 \mathrm{~kg} \mathrm{~m}^{-2}$. BMI was calculated as weight $(\mathrm{kg})$ divided by height $(\mathrm{m})^{2}$. Waist circumference(WC) was measured midway between the lowest rib and the iliac crest while the participants were standing upright. Bioelectrical impedance analysis (BIA) was used to estimate total fat mass, and percent body fat and fat mass of the trunk body $(\mathrm{kg})$ (Tanita bc-418 ma, Tokyo, Japan).

\section{Ultrasound examination}

All sonographic measurements were performed by a single radiologist with 15 years' experience on conventional ultrasonography (US). Subcutaneous fat(SCF) and preperitoneal fat (PPF) thickness measurements were done by using 5 to $12 \mathrm{MHz}$ linear transducer and visceral fat thickness measurements were done by using 3 to $5 \mathrm{MHz}$ convex array transducer with a Logic E9 ultrasound machine (GE Healthcare, Milwaukee, WI, USA).

\section{Abdominal fat measurements}

All the abdominal fat measurements were obtained in supine position by placing the probe perpendicular to the skin surface of the upper abdomen. Scanning was performed longitudinally from the xiphoid process to the umbilicus along the midline and measurements were obtained from the image captured when the probe touched lightly to the skin surface without applying a pressure. Subcutaneous fat is defined as the minimum distance from the cutaneous boundary to linea alba and preperitoneal fat is defined as the maximum distance between linea alba and the surface of the left lobe of the liver. Visceral fat thickness was measured as the distance between linea alba and the anterior aspect of the vertebral corpus. The measurement of visceral fat thickness was obtained $1 \mathrm{~cm}$ above the umbilicus on the imaginary line drawn between the xiphoid process and umbilicus. All the measurements were repeated three times and the average of these three measurements represented the final values of subcutaneous, preperitoneal and visceral fat thicknesses.

\section{Carotid intima - media thickness measurements}

Carotid intima - media thickness (CIMT) measurements were performed with a 5 to $12 \mathrm{MHz}$ linear transducer with the same US machine lying in supine position with a small cushion placed under the shoulders for extension of the neck and the neck rotated to opposite side of the examination. Common carotid artery (CCA) of the both sides were depicted in the longitudinal plane and measurements were obtained from the far wall of the CCA $2 \mathrm{~cm}$ proximal to the carotid bulb. Three measurements were performed from both CCAs with the automatic IMT measurement program of the ultrasound system. The average of these three measurements was recorded as the final value.

\section{Biochemical analyses}

Biochemical tests were performed on blood samples collected after overnight fasting. Serum glucose, triglyceride, total cholesterol and HDL cholesterol, creatinine, calcium, CRP levels and alanine transaminase activities were measured using the cobas c702 module of the Roche Cobas 8000 analyser in accordance with the manufacturer's instructions (Roche Diagnostics $\mathrm{GmbH}$, Penzburg, Germany). Fasting insulin and thyroid-stimulating hormone were measured by electrochemiluminescence immunoassays on the cobas e602 module of the Roche Cobas 8000 analyser in accordance with the manufacturer's instructions (Roche Diagnostics $\mathrm{GmbH}$, Penzburg, Germany). To determine the state of insulin resistance using the cut-off value of 2.7, the insulin-resistance homeostasis model assessment (HOMA) index was calculated using the following formula: HOMA-IR $=$ (glucose $(\mathrm{nmol} / \mathrm{L}) \times$ insulin $(\mu \mathrm{U} / \mathrm{ml}) / 22.5)$, using fasting values (Matthews et al., 1985). Plasma pentraxin-3 levels were measured by an enzyme-linked immunosorbent assay (ELISA) method (Hycult Biotech, Uden, The Netherlands) as follows. Samples and standards are incubated in microtiter wells coated with antibodies recognizing human pentraxin-3. Biotinylated tracer antibody binds to captured human pentraxin-3. Streptavidin-peroxidase conjugate binds to the biotinylated tracer antibody, and reacts with the substrate, tetramethyl benzidine. The enzyme reaction is stopped by the addition of oxalic acid. The absorbance at $450 \mathrm{~nm}$ is measured with multiplate ELISA reader (DAS, Digital and Analog Systems, Vimercate, MI, Italy). 


\section{Statistical analyses}

Statistical analysis was performed using SPSS v.18.0 for Windows (SPSS, Inc., Chicago, IL). Continuous and categorical data are shown as mean $\pm S D$ and percentage, respectively. When parametric test assumptions were provided, independent samples $t$ test were used for intergroup comparisons. If assumptions were not provided, Mann Whitney $U$ test were used. Correlation between continuous variables were analysed with Spearman Correlation Coefficient. Multiple linear regression analysis was used to identify the factors associated with osteocalcin. The level of statistical significance was set at $p<0.05$.

\section{RESULTS}

The antropometric measures of subjects are shown in Table 1. Obese subjects had increased adiposity indices such as BMI, waist circumference, body fat mass, VF, PPF and SCF compared to normal weight subjects ( $p<$ 0.01 ). Biochemical and hormonal variables of obese and controls are shown in Table 2.

PTX3 was similar in both groups. PTX3 was also similar in obese patients with or without insulin resistance. CRP is significantly higher in obese patients $(p<0.01)$. It was also significantly higher in insulin resistance obese patients $(p<0.01)$. PTX3 is correlated only with ALT ( $p=0.033, r=+0.250)$ There was no correlation between BMI or any of the inflammatory markers. Multiple regression analysis showed that PTX3 is positively correlated with WC $(p=0.036$, beta $=$ $+0.436)$ and negatively correlated with SCF $(p=0.11$, beta $=-0.310)$.

\section{DISCUSSION}

In the present study, we found a positive correlation between serum levels of PTX3 and WC. Pentraxin 3 level is also negatively associated with SCF but not correlated with VF and PPF after adjustment with age, sex, other inflammatory markers, glucose levels and HOMA in premenopausal obese women.

Studies showed that obese people have elevated plasma PTX3 levels (Miyaki et al., 2013) and an increased expression of visceral adipose tissue PTX3 that associates with a cardiovascular risk profile, including low HDL-cholesterol and high fibrinogen (Alberti et al., 2009). Increased PTX3 concentrations were also reported in individuals with a metabolic syndrome phenotype (Zanetti et al., 2009). These initial findings, indicate that PTX3 production might be upregulated in obesity and possibly playing a role in CVD. But this has been challenged by several independent studies that demonstrate an inverse relation between body mass and systemic PTX3 concentrations (Osorio-Conles et al., 2011; Barazzoni et al., 2013; Miyaki et al., 2013; Miyamoto et al., 2011; Ogawa et al., 2010; Yamasaki et al., 2009). In our study PTX3 did not seem to be elevated in premenopausal obese women.

Bosutti et al. (2008) demonstrated that plasma PTX3 levels inversely correlated with fat mass. Ogawa et al. found that PTX3 correlated negatively with body weight, $\mathrm{BMI}, \mathrm{WC}$, triglyceride and positively with adiponectin. Plasma CRP significantly increased, whereas plasma PTX3 significantly decreased with increasing BMI. Plasma CRP levels were significantly higher and PTX3 levels were significantly lower in individuals who had more than one metabolic syndrome (MetS) component compared with those who had none. They concluded that PTX3 and CRP antagonistically participate in the development of obesity or MetS (Ogawa et al., 2010). Two studies reporting positive associations between PTX3 and BMI included obese subjects (Alberti et al., 2009; Miyaki et al., 2010), whereas the studies reporting inverse associations were reported primarily in overweight subjects (Osorio-Conles et al., 2011). Altough the mean BMI was 38.3 in our study still there was no association between BMI and PTX3. There is no clear explanation for these discrepancies. It is tempting to hypothesize that the multiple sources of PTX3 may serve different functions according the tissue or the cellular component where it is produced. Because PTX 3 is being produced by cells at the inflammatory site, circulating PTX3 levels may not fully reflect the molecular activities in peripheral tissues such as adipose tissue. Thus, it is of interest to identify local effects that may occur via autocrine/paracrine pathways in the adipose tissue. Witasp et al. also showed that visceral, but not subcutaneous adipose tissue, is associated with PTX3 serum levels (Witasp et al., 2014). This may highlight an important pathogenic link as visceral adipose tissue PTX3 gene expression has been associated with cardiovascular risk factors (Alberti et al., 2009). In our study, we found that PTX was positively correlated with WC and inversely associated with SCF, although we could not find any correlation with VF, PPF in obese premenopasual women. Different study populations may be one of the reasons for the different results. Further, it supports the need for additional studies to describe the reasonable adipose tissue-specific contribution to circulating PTX3 levels and its relation to cardiometabolic risk factors and body fat distribution.

The role of PTX3 as a biomarker of cardiovascular inflammation and progression independently of other acute phase proteins such as CRP was studied in the Bruneck Study (Kiechl et al., 2001), PTX3 levels were higher in individuals with atherosclerotic plaques and prevalent vascular diseases, but not correlated with the CIMT which is considered a marker of subclinical atherosclerosis. In patients with MetS, PTX3 levels were directly correlated with CIMT (Zanetti et al., 2009); this correlation, however, was no longer significant after adjustment for HDL-C levels. While a cross-sectional analysis showed an independent association between PTX3 plasma levels and advanced human 
Table 1. Antropometric measures and fat distrubution of obese and controls.

\begin{tabular}{lccc}
\hline Parameter & Obese $(\mathbf{n}=\mathbf{7 3})$ & Control $(\mathbf{n}=\mathbf{5 3})$ & $\mathbf{P}$ \\
\hline Weight $(\mathrm{kg})$ & $97.2 \pm 15.8$ & $61.0 \pm 10.1$ & $0.000^{\star}$ \\
BMl & $38.3 \pm 5.4$ & $23.5 \pm 3.5$ & $0.000^{\star}$ \\
WC $(\mathrm{cm})$ & $107.4 \pm 11.3$ & $80.0 \pm 9.8$ & $0.000^{\star}$ \\
Trunckal fat percent & $38.7 \pm 5.1$ & $20.4 \pm 8.9$ & $0.000^{*}$ \\
Total fat mass $(\mathrm{kg})$ & $42.9 \pm 10.5$ & $16.7 \pm 6.7$ & $0.000^{*}$ \\
Percent body fat & $43.5 \pm 4.2$ & $26.5 \pm 7.1$ & $0.000^{\star}$ \\
VF $(\mathrm{mm})$ & $54.2 \pm 22.3$ & $30.0 \pm 11.3$ & $0.000^{\star}$ \\
PPF $(\mathrm{mm})$ & $20.4 \pm 30.5$ & $6.7 \pm 3.1$ & $0.000^{\star}$ \\
SCF $(\mathrm{mm})$ & $34.4 \pm 10.5$ & $18.6 \pm 8.5$ & $0.000^{\star}$ \\
CIMT $(\mathrm{mm})$ & $1.4 \pm 2.0$ & $0.5 \pm 0.1$ & $0.000^{\star}$ \\
\hline
\end{tabular}

BMI: Body mass index; WC: Waist circumference; VF: Visseral fat; PPF: Preperitoneal fat; SCF: Subcutanous fat; CIMT:Carotis intima media thickness.

Table 2. Biochemical and hormonal variables of obese and controls.

\begin{tabular}{lccc}
\hline Parameter & Obese $(\mathbf{n}=\mathbf{7 3})$ & Control $(\mathbf{n}=\mathbf{5 3})$ & $\mathbf{P}$ \\
\hline FBG $(\mathrm{mg} / \mathrm{dl})$ & $96.3 \pm 10.8$ & $89.6 \pm 9.0$ & $0.000^{*}$ \\
Insulin (ulU/ml) & $19.1 \pm 10.4$ & $9.1 \pm 6.5$ & $0.000^{*}$ \\
HOMA & $4.6 \pm 2.8$ & $2.0 \pm 1.9$ & $0.000^{*}$ \\
Creatinine (mg/dl) & $0.6 \pm 0.1$ & $0.6 \pm 0.1$ & 0.718 \\
ALT ( IU/L) & $20.2 \pm 11.1$ & $16.0 \pm 9.6$ & $0.001^{*}$ \\
LDL (mg/dl) & $106.3 \pm 11.1$ & $93.3 \pm 9.6$ & $0.013^{*}$ \\
HDL (mg/dl) & $50.5 \pm 11.2$ & $66.5 \pm 14.3$ & $0.000^{*}$ \\
TG (mg/dl) & $116.3 \pm 54.8$ & $85.2 \pm 48.0$ & $0.000^{*}$ \\
TSH (ulU/ml) & $3.0 \pm 1.3$ & $1.9 \pm 1.2$ & $0.020^{*}$ \\
Calcium (mg/dl) & $9.1 \pm 0.5$ & $9.1 \pm 0.4$ & 0.385 \\
PTH (pg/ml) & $61.9 \pm 24.7$ & $52.5 \pm 18.4$ & $0.049^{*}$ \\
Vitamin D (ng/ml) & $13.4 \pm 9.9$ & $14.0 \pm 11.0$ & 0.433 \\
Phosphorus (mg/dl) & $3.2 \pm 0.6$ & $3.3 \pm 0.4$ & 0.170 \\
Magnesium (mg/dl) & $1.9 \pm 0.1$ & $1.9 \pm 0.2$ & $0.022^{*}$ \\
ALP $(\mathrm{IU} / \mathrm{L})$ & $77.1 \pm 25.2$ & $55.3 \pm 16.6$ & $0.000^{*}$ \\
PT3 (pg/ml) & $0.8 \pm 0.6$ & $0.9 \pm 0.8$ & 0,785 \\
\hline
\end{tabular}

${ }^{*} \mathrm{p}<0.05$ statistically significant. FBG: Fasting blood glucose; ALT: Alanine transaminase; LDL: Low density lipoprotein; HDL: High density lipoprtptein; TG: Triglycerides; TSH: Thyroid stimulating hormone; PT3: Pentraxin3; CRP: C-reaktive protein.

atherosclerosis (Knoflach et al., 2012), the prospective analysis failed to suggest a correlation of PTX3 plasma levels with the progression of subclinical atherosclerosis and the incidence of vascular damage as measured by CIMT in two large cohorts (Baragetti et al., 2014). In our study, pentraxin was not correlated with CIMT in premenopausal obese women.

\section{Conclusion}

Although PTX3 levels were not higher in obese patients and has no correlation with visceral fat, it is correlated with WC. In premenopausal obese women PTX3 levels were not related to subclinical atherosclerosis measured by CIMT. To clarify this further studies are needed.

\section{ACKNOWLEDGEMENT}

This study is funded by Pamukkale University Scientific Research Project Coordiantion Unit through Project number 2014TPF048.

The authors declare that there is no conflict of interests regarding the publication of this article.

\section{REFERENCES}

Abderrahim-Ferkoune A, Bezy O, Chiellini C, Maffei $\quad M$, Grimaldi P, Bonino F, Moustaid-Moussa N, Pasqualini F, Mantovani A, Ailhaud 
G, Amri E, 2003. Characterization of thelong pentraxin PTX3 as a TNFa-induced secreted protein of adipose cells. J Lipid Res, 44:9941000.

Alberti L, Gilardini L, Zulian A, Micheletto G, Peri G, Doni A, Mantovani A, Invitti C, 2009. Expression of long pentraxin PTX3 in human adipose tissue and its relation with cardiovascular risk factors. Atherosclerosis, 202: 455-460.

Baragetti A, Knoflach M, Cuccovillo I, Grigore L, Casula M, Garlaschelli $K$, 2014. Pentraxin 3 (PTX3) plasma levels and carotid intima media thickness progression in the general population. Nutr Metab Cardiovasc Dis, 24(5): 518-523.

Barazzoni R, Aleksova A, Carriere C, Cattin MR, Zanetti M, Vinci P, Stolfo D, Guarnieri G, Sinagra G, 2013. Obesity and high waist circumference are associated with low circulating pentraxin-3 in acute coronary syndrome. Cardiovasc Diabetol,12: 167.

Bonacina F, Baragetti A, Catapano AL, Norata GD, 2013. Long pentraxin 3: experimental and clinical relevance in cardiovascular diseases. Mediators Inflamm, 2013:10.

Bosutti A, Malaponte G, Zanetti M, Castellino P, Heer M, Guarnieri G, Biolo G, 2008. Calorie restriction modulates inactivity-induced changes in the inflammatory markers C-reactive proteinand pentraxin-3. J Clin Endocrinol Metab, 93: 3226-3229.

Garlanda C, Bottazzi B, Bastone A, Mantovani A, 2005. Pentraxins at the crossroads between innate immunity, inflammation, matrix deposition, andfemale fertility. Annu Rev Immunol, 23: 337-366.

Kiechl S, Egger G, Mayr M, Wiedermann CJ, Bonora E, Oberhollenzer F, Muggeo M, Xu Q, Wick G, Poewe W, Willeit J, 2001. Chronic infections and the risk of carotid atherosclerosis: prospective results from a large population study. Circulation, 103: 1064-1070.

Knoflach M, Kiechl S, Kind M, Said M, Sief R, Gisinger M, van der Zee R, Gaston H, Jarosch E, Willeit J, Wick G, 2003. Cardiovascular risk factors and atherosclerosis in young males: ARMY study (Atherosclerosis Risk-Factors in Male Youngsters). Circulation, 108: 1064-1069.

Knoflach M, Kiechl S, Mantovani A, Cuccovillo I, Bottazzi B, Xu Q, Xiao Q, Gasperi A, Mayr A, Kehrer M, Willeit J, Wick G, 2012. Pentraxin-3 as a marker of advanced atherosclerosis results from the Bruneck, ARMY and ARFY studies. PLoS One, 7:e31474.

Knoflach M, Kiechl S, Penz D, Zangerle A, Schmidauer C, Rossmann A, Shingh M, Spallek R, Griesmacher A, Bernhard D, Robatscher P, Buchberger W, Draxl W, Willeit J, Wick G, 2009. Cardiovascular risk factors and atherosclerosis in young women: atherosclerosis risk factors in female youngsters (ARFY study). Stroke, 40:1063-1069.

Matthews DR, Hosker JP, Rudenski AS, Naylor BA, Treacher DF, Turner RC, 1985. Homeostasis model assessment: insulin resistance and beta-cell function from fasting plasma glucose and insulin concentrations in man. Diabetologia, 28: 412-419.

Miyaki A, Maeda S, Choi Y, Akazawa N, Eto M, Tanaka K, Ajisaka R, 2013. Association of plasma pentraxin 3 with arterial stiffness in overweight and obese individuals. Am J Hypertens, 26: 1250-1255.

Miyaki A, Maeda S, Yoshizawa M, Misono M, Sasai H, Shimojo $\mathrm{N}$, Tanaka K, Ajisaka R, 2010. Is pentraxin 3 involved in obesityinduced decrease in arterial distensibility? J Atheroscler Thromb, 17: 278-284.

Miyamoto $T$, Rashid Qureshi A, Heimburger $O$, Bárány $P$, Carrero K, Sjöberg B, Lindholm B, Stenvinkel $P$, Carrero JJ, 2011. Inverse relationship between the inflammatory marker pentraxin-3, fat body mass, and abdominal obesity in endstage renal disease. Clin $\mathrm{J} \mathrm{Am}$ Soc Nephrol, 6: 2785-2791.

Ogawa T, Kawano Y, Imamura T, Kawakita K, Sagara M, Matsuo T, Kakitsubata $Y$, Ishikawa T, Kitamura K, Hatakeyama K, Asada $Y$, Kodama T, 2010. Reciprocal contribution of pentraxin 3 and Creactive protein to obesity and metabolic syndrome. Obesity (Silver Spring), 18: 1871-1874.

Osorio-Conles O, Guitart M, Chacón MR, Maymo-Masip E, MorenoNavarrete JM, Montori-Grau M, Näf S, Fernandez-Real JM, Vendrell J, Gómez-Foix AM, 2011. Plasma PTX3 protein levels inversely correlate with insulin secretion and obesity, whereas visceral adipose tissue PTX3 gene expression is increased in obesity. Am J Physiol Endocrinol Metab, 301:E1254-E1261.

Pepys MB, Hirschfield GM, 2003. C-reactive protein: a critical update. J Clin Invest, 111: 1805-1812.
Peri G, Introna M, Corradi D, lacuitti G, Signorini S, Avanzini F, Pizzetti F, Maggioni AP, Moccetti T, Metra M, Cas LD, Ghezzi P, Sipe JD, Re G, Olivetti G, Mantovani A, Latini R, 2000. PTX3, a prototypical long pentraxin, is an early indicator ofacute myocardial infarction in humans. Circulation, 102: 636e41.

Ridker PM, Hennekens CH, Buring JE, Rifai N, 2000. C-reactive protein andother markers of inflammation in the prediction of cardiovascular disease inwomen. N Engl J Med, 342: 836-843.

Witasp A, Carrero JJ, Michaëlsson K, Ahlström $H$, Kullberg $\mathrm{J}$, Adamsson V, Risérus U, Larsson A, Helmersson-Karlqvist J, Lind L, Stenvinkel P, Arnlöv J, 2014. Inflammatory biomarker pentraxin 3 (PTX3) in relation to obesity, body fat depots and weight loss. Obesity (Silver Spring), 22(5): 1373-1379.

Yamasaki K, Kurimura M, Kasai T, Sagara M, Kodama T, Inoue K, 2009. Determination of physiological plasma pentraxin 3 (PTX3) levels in healthy populations. Clin Chem Lab Med, 47: 471-477.

Zanetti M, Bosutti A, Ferreira C, Vinci P, Biolo G, Fonda M, Valente M, Cattin L, Guarnieri G, Barazzoni R, 2009. Circulating pentraxin 3 levels are higher in metabolic syndrome with subclinical atherosclerosis: evidence for association with atherogenic lipid profile. Clin Exp Med, 9: 243-248.

Citation: Dedeoglu O, Yaylali GF' Herek D' Demir S, Senol H, Topsakal $\mathrm{S}, 2018$. Association between pentraxin-3, body fat distribution and carotid intima media thickness in premenopausal obese women. Int Res J Med Med Sci, 7(1): 16-20. 